\title{
Studies in auditory timing: 1. Simple patterns
}

\author{
IRA J. HIRSH, CAROLINE B. MONAHAN, KEN W. GRANT, and PUNITA G. SINGH \\ Central Institute for the Deaf, St. Louis, Missouri
}

\begin{abstract}
Listeners' accuracy in discriminating one temporal pattern from another was measured in three psychophysical experiments. When the standard pattern consisted of equally timed (isochronic) brief tones, whose interonset intervals (IOIs) were 50,100, or $200 \mathrm{msec}$, the accuracy in detecting an asynchrony or deviation of one tone in the sequence was about as would be predicted from older research on the discrimination of single time intervals $(\theta \%-8 \%$ at an IOI of $200 \mathrm{msec}, 11 \%-12 \%$ at an IOI of $100 \mathrm{msec}$, and almost $20 \%$ at an IOI of $50 \mathrm{msec}$ ). In a series of 6 or 10 tones, this accuracy was independent of position of delay for IOIs of 100 and $200 \mathrm{msec}$. At $50 \mathrm{msec}$, however, accuracy depended on position, being worst in initial positions and best in final positions. When one tone in a series of six has a frequency different from the others, there is some evidence (at $\mathrm{IOI}=200 \mathrm{msec}$ ) that interval discrimination is relatively poorer for the tone with the different frequency. Similarly, even if all tones have the same frequency but one interval in the series is made twice as long as the others, temporal discrimination is poorer for the tones bordering the longer interval, although this result is dependent on tempo or IOI. Results with these temporally more complex patterns may be interpreted in part by applying the relative Weber ratio to the intervals before and after the delayed tone. Alternatively, these experiments may show the influence of accent on the temporal discrimination of individual tones.
\end{abstract}

Temporal aspects of auditory perception, including observations on recognition and discrimination of rhythmic patterns as well as models and theories of timing and rhythmic groups, have recently become the subject of a rich body of literature, particularly in music perception. Different studies have emphasized different aspectspreference for certain phrases, recognition of melodic segments, imitative tapping to infer a "representation" of patterns, and so forth. Our interest here, somewhat less grand, concerns listeners' accuracy in the discrimination of temporal patterns.

The timing of successive elements in an auditory pattern is critical for the identification both of particular sounds and of characteristics of patterns as a whole. The perception of stress in speech or of accent and rhythmic structure in music requires at least that listeners can discriminate different dimensions of individual sounds, including duration, and also different time intervals separating the onsets of successive sounds. The classical literature on temporal discrimination (Woodrow, 1951) suggests that for a reasonably large range of standard time values, discrimination is reliably good when deviations from the standard are of the order of $10 \%$. Woodrow's chapter dis-

This research was supported by a grant from the U.S. Air Force Office of Scientific Research to the Central Institute for the Deaf, and by Washington University, where Ira Hirsh is a Distinguished University Professor. Thanks are due to Stephen Handel and an anonymous reviewer for helpful suggestions on the manuscript. Ken Grant is now at the Massachusetts Institute of Technology. Correspondence may be addressed to I. J. Hirsh or Caroline B. Monahan, Central Institute for the Deaf, 818 South Euclid, St. Louis, MO 63110. tinguishes clearly between two types of intervals used in studies on temporal discrimination: (1) empty intervals, or the time intervening between two boundary events, whether sounds or lights, and (2) continuous stimuli, in which judgments are made about the apparent duration of the events themselves. Woodrow quotes a 1933 study of Blakely as indicating that empty-interval discrimination was just under $10 \%$ in the range of intervals from 200 to $1,500 \mathrm{msec}$, and worsened to $16 \%-20 \%$ as the standard interval increased beyond $2,000 \mathrm{msec}$. Similar results are quoted from a 1933 study by Stott for the discrimination of continuous-sound durations. These findings were approximately confirmed by Small and Campbell (1962) for the range $40-400 \mathrm{msec}$, by Creelman (1962) for the range 100-1,000 msec, and by Abel (1972) for the range $50-500 \mathrm{msec}$. The $10 \%$ figure is essentially a constant Weber ratio for durations and intervals between about 200 and $2,000 \mathrm{msec}$.

Applications of such basic psychophysical data to rhythmic aspects of speech and music may well be direct, but it does not appear that typical speech and music listening places demands upon the listener that come even close to these capabilities. (We speak of syllables and notes, and not the fine temporal grain that concerns individual speech sounds or onsets of musical tones.) In music, note durations are typically changed by $100 \%$ (upward from quarter to half to whole, etc.). In English speech, the duration of stressed as opposed to unstressed syllables is on the order of $150 \%$ (Fry, 1955; Umeda, 1975), as is the lengthening of a vowel associated with a following voiced as opposed to an unvoiced consonant. These speech and music examples involve both empty and filled intervals. 
As one lengthens notes in a melody, both note durations and timing, or note-to-note onsets, are increased. In the rhythmic patterns of successions of stressed and unstressed syllables in poetry or the rhythmic structures of musical sequences, both the single-sound duration and the soundto-sound timing are important ingredients.

The present experiments were motivated by an interest in the range of applicability of temporal psychophysical data to the complex contexts of longer sequences. In the older studies, temporal discrimination was measured with two isolated events to be compared in apparent duration, or with two successive intervals, each bounded by a pair of stimulus events. Is temporal discrimination comparable in cases in which two such sounds or two such intervals are part of a longer sequence?

The applicability of temporal psychophysical data to the longer sequences of speech and music involves duration discrimination, timing discrimination, detection of deviations from time markers in sound sequences, and changes in timing at different positions within the sequence. The possible influence of the pitch, timbre, or loudness of particular tones in a sequence on the accuracy of perceiving the time of occurrence or the duration of such tones or of neighboring tones can be further complicated, as in the interaction between frequency changes and temporal discrimination in nonisochronic patterns (Sorkin, 1987; Sorkin, Boggs, \& Brady, 1982). These features are similar to some studied by Watson, Wroton, Kelly, and Benbassat (1975) for relatively fast tempos of event succession.

Another set of questions concerning the applicability of psychophysical data to sequences of speech and music concerns linguistic and musical rules. Such concepts as phrases, stress, and prosodic form in language, or rhythmic structure, accent, metricality, and the various time signatures in music, invoke a more cognitive aspect that may well influence the role played by timing in these two subsets of sequential patterns.

\section{PILOT STUDIES}

\section{Duration Discrimination}

To repeat some classical experiments in which listeners are asked to discriminate between long and short durations, we presented pairs of short (S) and long (L) tones, where $S$ was 25 or $200 \mathrm{msec}$ and the values of $\mathrm{L} / \mathrm{S}$ were 1.3 , $1.2,1.1,1.05$, and 1.025. The timing was always constant; the tone-pulse onsets in each pair were separated by $320 \mathrm{msec}$. Discriminations (same vs. different) could not be based on timing intervals separating onsets. Musically speaking, we have a "staccato" versus a "legato", at a constant tempo, and thus an example of what are classically labeled "filled intervals."

Interpolated values of $L / S$ for 3 subjects for $d^{\prime}=1$ (about $70 \%$ correct) showed increments typical of the past-namely, about $8 \%$ at $200 \mathrm{msec}$ and $15 \%-20 \%$ at $25 \mathrm{msec}$. When the task was changed to one of identifying one of the four possible 2-tone patterns (i.e., LL, SS, SL, LS), performance was not very different. Perhaps we might as well have asked for identification as trochees, iambs, or spondees (see Vos, 1977), but in the case of those stress patterns, differences are in both duration and timing.

A first step toward sequential patterns involved presenting two such pairs, the first or second of which contained two equal durations, while the other contained either SL or LS. Thus, we had four different 4-tone patterns, with the first pair being separated from the second pair by an additional $320 \mathrm{msec}$ (i.e., SS-SL, SS-LS, SL-SS, LS-SS). Listeners had to identify which of the 4 tones was L. This 4AFC procedure yielded results that were quite similar to those for the same patterns (in which there were three Ss and one L) under a different task in which the listener had to identify which 4-tone pattern was presented. In this case, the difference between a 4IFC choice and the identification was trivial. These pattern sets were also repeated without any additional gap between the first and second pairs, again with no big differences.

We also tried cases in which the 4-tone patterns consisted of two Ss and two Ls, the differences among the patterns consisting principally of the order of elements that were perceived to be long or short. In the first case, we asked the listener to tell whether the first pair of tones was the same or different from the second pair of tones. This judgment is relatively complex, requiring a recognition of a difference in duration and the comparison of the order of two elements, judged to be different in duration, within each of the pairs. The discrimination task now required comparison between two pairs resulting in somewhat higher difference limens (DLs). We did not pursue these differences because it appeared that performance for duration discrimination was not remarkably different when the tones were embedded in multitone patterns as compared with simple tone pairs. In fact, Henry (1948) had shown that a pattern of alternating tones of equal duration could be discriminated from one in which alternation was between unequal durations, so long as the durations were different by about $10 \%$.

\section{Timing Discrimination}

In the next series of experiments, the duration of the individual sounds within the pattern was held constant, while the intervals separating their onsets varied. In the first experiment, we studied the ability of listeners to detect a deviation from regular, periodic timing of a single element in a series of 3,6 , or 10 tones with the standard interevent or interonset interval (IOI) held at 50, 200, or $800 \mathrm{msec}$, corresponding to rates or tempos of 20,5 , and 1.25 tones per second, respectively. The subjects were asked to indicate whether two successive patterns were the same or different. Using both a same-different procedure and an adaptive procedure, in which correct responses automatically decreased the amount by which one of the tones had been delayed within an otherwise isochronic series, we found that, at standard intervals of 200 and $800 \mathrm{msec}$, a constant value of 5\%-8\% represents the data reasonably well and seems to be independent of the posi- 
tion in the pattern where the element was offset. For the fastest series, with an IOI of $50 \mathrm{msec}$, there was an overall increase in the offset (in milliseconds) required for discrimination and, in addition, a dependence of the results on the position of the element offset in the sequence. In a 6- or 10-tone pattern, when a delay was introduced on the second or third tone, that delay had to be greater for pattern discrimination than when the delay was introduced for a later tone. This result is similar to the recency effect for frequency discrimination in the studies of Watson and his colleagues (1975) for series of tones with an IOI of $40 \mathrm{msec}$. It appears that phenomena observable for intervals of less than $100 \mathrm{msec}$ might obey a different set of rules from those that obtain for longer intervals (Pollack, 1967).

Changes in the elements. The dependence of delay discrimination on position of the delayed tone within the pattern was the first of a series of earlier studies on withinpattern features. The independent variable was a feature describing the role of a tone in a pattern, as distinguished from a feature of the tone itself. In music, rhythmic changes often involve not only timing changes but also changes in the pitches of a melody. We presented the same groups of 6 tones at an IOI of $200 \mathrm{msec}$, but then introduced a single downward frequency change from 1000 to $600 \mathrm{~Hz}$, or an upward change from 1000 to $1667 \mathrm{~Hz}$ (frequency ratio of 5/3) at Positions 2, 3, or 5. Comparison of the results for a delayed tone at Positions 2, 3, or 5 showed clearly that a delay in the tone that also has a frequency shift is more difficult to discriminate from isochrony than is a delay in the other positions. The effect was similar for the downward and the upward shifts, but appeared to be more pronounced for the downward shifts. Temporal discrimination was poorest for the position that coincides with the tone whose pitch was changed. This result is consistent with previous findings in our laboratory (Divenyi \& Danner, 1977), and also with those of Fitzgibbons, Pollatsek, and Thomas (1974) concerning the discrimination of a temporal gap between two sounds that differ in spectrum.

\section{EXPERIMENT 1 DELAY OF A TONE IN A SIMPLE SERIES}

The pilot studies (Hirsh, 1987) suggested that the discrimination of an isochronic, monotone sequence from another in which one of the tonal elements was delayed was as good for IOIs of 200 and $800 \mathrm{msec}$ as it would have been in the discrimination of single intervals bounded by those tones. Furthermore, this excellent performance was equally good across all positions in the sequence. At IOIs of $50 \mathrm{msec}$, discrimination performance was much poorer and showed a marked improvement as the position of the delayed tone was advanced toward the end of the pattern. In the first experiment, we sought to repeat those observations.

\section{Overview of the Design}

Two different experiments (1A and $1 \mathrm{~B}$ ) employed isochronic series of 6 and 10 tones, respectively, as standards. Both experiments comprised complete repeated measure designs with the same three factors: tempo (IOIs of 200,100 , or $50 \mathrm{msec}$ between tones), position of tone delay (Tone Positions 2, 3, and 5 in Experiment 1A, and Positions 2, 3, 5, and 9 in Experiment 1B), and number of replications (three). Thus, there were nine conditions for each replication of Experiment $1 \mathrm{~A}$ and 12 conditions for each replication of Experiment 1B. All tones in these patterns were $1000-\mathrm{Hz}$ sinusoids, $20 \mathrm{msec}$ in duration (2.5-msec rise-fall times plus $15 \mathrm{msec}$ sustained).

\section{Method}

Apparatus. A DEC PDP-11/73 computer controlled the experiments. Sinusoidal tones were generated digitally $(20,000$ samples/ $\mathrm{sec}$ ), passed to a D/A converter, filtered, and sent to earphones in a quiet room. Steady-state equivalent sound level at the earphones was maintained at $80.5 \mathrm{~dB} \mathrm{SPL} \pm 0.2 \mathrm{~dB}$ (artificial ear). Voltage to the earphones was calibrated daily. The computer collected subject responses and stored them in data files along with stimulus and timing parameters for each trial.

Subjects. There were 3 female subjects, all with normal audiograms. Subjects H.D., S.K., and J.K. had had 4, 9, and 10 years, of musical experience, respectively. J.K. was a self-run subject and served as experimenter for most of the conditions for the other 2 subjects. All subjects were highly practiced with the procedures of the present experiment, having previously taken part in two or more similar experiments. The subjects were paid for their services.

Procedure. Before data were taken, the subjects performed nine adaptive runs, corresponding to the experimental conditions of the 6-tone experiment, as practice. At the beginning of each run, the experimenter entered the tempo and serial position of the tone to be delayed into the adaptive program, and told the subject the serial position of the tone to be delayed. The experimenter then played the standard followed by a comparison sequence with a delay well above threshold, and ascertained that the subject could hear a difference between the sequences.

The experimenter then initiated the cued $2 \mathrm{AFC}$ procedure in which each trial comprised a standard and two comparison patterns, one of which, at random, had the delayed tone. Each run started with a delay well above the discrimination threshold $(50 \%$ of the standard IOI). Thus, at the medium tempo, the initial trial with a delay of the third tone would take the form shown in Figure 1, although the order of the two ccmparisons might be reversed (sound-filled and silent portions are shown for each IOI in milliseconds). We emphasize that the single delay of a tone always involved a lengthening of the preceding interval and a consequent shortening of the following interval, so that the timing continued (except for the tone delayed) and the length of the pattern remained the same.

On each trial, the subject indicated which of the two comparison sequences contained the delay by pushing one of two keys on a computer terminal. The response interval was $3 \mathrm{sec}$; if the subject failed to respond in that time, she was prompted to answer by a message on the terminal screen until a response was given. After each response, the terminal screen displayed feedback. In the adaptive procedure, for every two correct answers, the relative (percentage) delay was halved; for every incorrect answer, the relative delay was doubled. The completion of each run required five reversals (an "up" followed by a "down" trial, or a "down" trial followed by an "up" trial) in the amount of detectable delay; each run gener- 
STRUCTURE OF TRIAL

EXAMPLE: PATTERN 11111 WITH DELAY OF THIRD TONE STANDARD

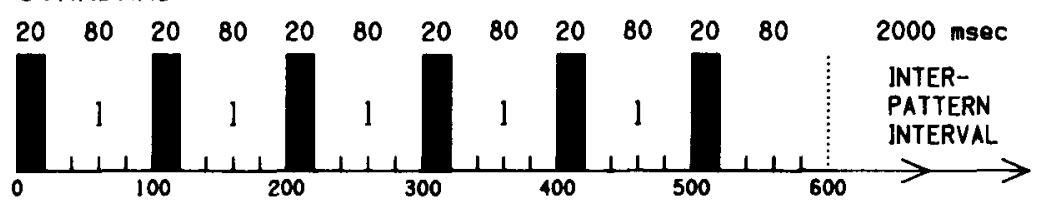

COMPARISON 1
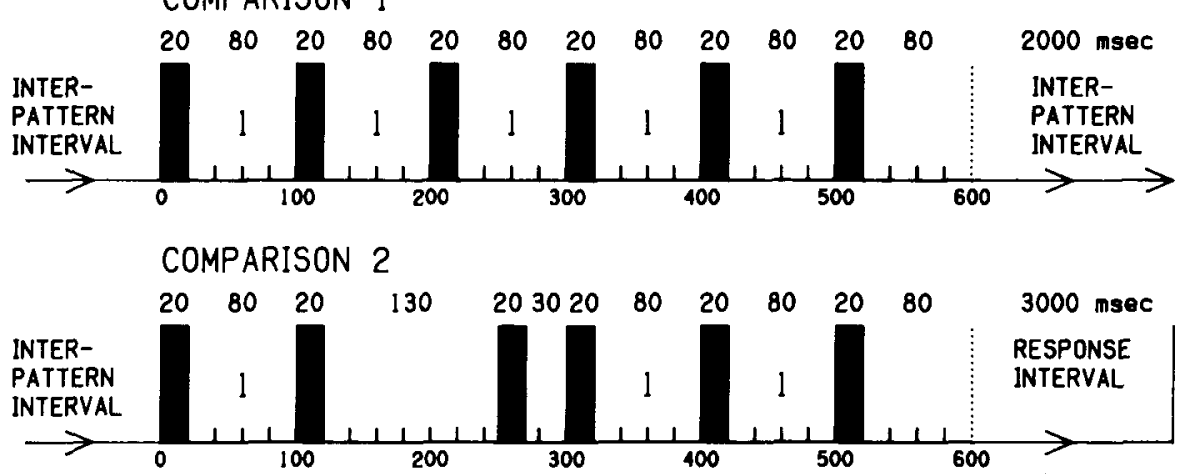

Figure 1. Timing diagram for Experiment 1.

ally took from 12 to 25 trials. The mean delay and standard deviation (in percent of the IOI, $\Delta t / t \times 100$ ) for the last 10 trials of each run were computed and stored.

The 6-tone experiment was run prior to the 10-tone experiment. The 9 conditions of Experiment $1 \mathrm{~A}$ and the 12 conditions of Experiment $1 \mathrm{~B}$ were presented in different random orders for each replication for each subject. For each subject, all 9 or 12 conditions of any particular replication were completed before the next replication was begun. There were, in fact, four replications, but the analyses were carried out on only the last three. The subjects completed each experiment in a period of about 2 weeks; experimental sessions lasted between 1 and $2 \mathrm{~h}$ with 10 to 20 adaptive runs per session.

\section{Results}

Two analyses were performed. For Experiment 1A, a repeated measures analysis of variance (ANOVA) (3 tempos $\times 3$ positions of delay $\times 3$ replications) was run on the estimates of the Weber ratio for discrimination of delay for the conditions of the 6-tone experiment. The only significant main effect was that of tempo $[F(2,4)=58.95$, $p<.002]$. The Weber ratios $(\times 100)$ for the slow, medium, and fast tempos were 5.66, 11.66, and 20.76, respectively. The means for the nine conditions of the 6-tone experiment $(3$ tempos $\times 3$ positions of delayed tone) summed over subjects and replications are shown in Figure 2A. (In both panels, the standard error of each mean represents variation across subjects and is based on an $n$ of 3. Standard errors less than 1 Weber-ratio unit are not shown.)

For Experiment 1B, a repeated measures ANOVA ( 3 tempos $\times 4$ positions of delay $\times 3$ replications) was run on the estimates of the Weber ratio for delay discrimination in the 10-tone experiment. The only significant main effect was that of tempo $[F(2,4)=24.73, p<.006]$. The Weber ratios $(\times 100)$ for the slow, medium, and fast tempos were $6.2,11.33$, and 18.46 , respectively. In addition, the interaction of tempo and position of delay approached significance $[F(6,12)=2.74, p<.065]$. This result reflects the greater difficulty that the subjects had in detecting delays of the second tone in the fast-tempo condition relative to the other tempo conditions. The means for the 12 conditions of the 10-tone experiment ( 3 tempos $\times 4$ positions of delayed tone), summed over subjects and replications, are shown in Figure 2B.

These results confirm their counterparts in the pilot studies and show, as well, levels of discrimination comparable to more classical data. At slow tempos (IOI = $200 \mathrm{msec}$, or $5 \mathrm{tones} / \mathrm{sec}$ ), the relative DL for intervals is about $5 \%-6 \%$, and holds equally for the delay of any tone in the series. Only when the tempo was fast (IOI = $50 \mathrm{msec}$, or 20 tones $/ \mathrm{sec}$ ) and the discrimination was poorer (DL between 15\% and 30\%) does there appear to be a dependence of interval discrimination on position in the pattern. At fast tempos, temporal discrimination for the earliest elements was poor.

\section{EXPERIMENT 2 TONE SERIES WITH PITCH CHANGE}

When a sequence of tones comprises a pattern, we may speak of two classes of properties or features of the tones. One is intrinsic and belongs to the tone itself. Another is based upon the role played by the tone in the sequence. In Experiment 1, one independent variable, which turned out to be an important predictor of discrimination at fast 

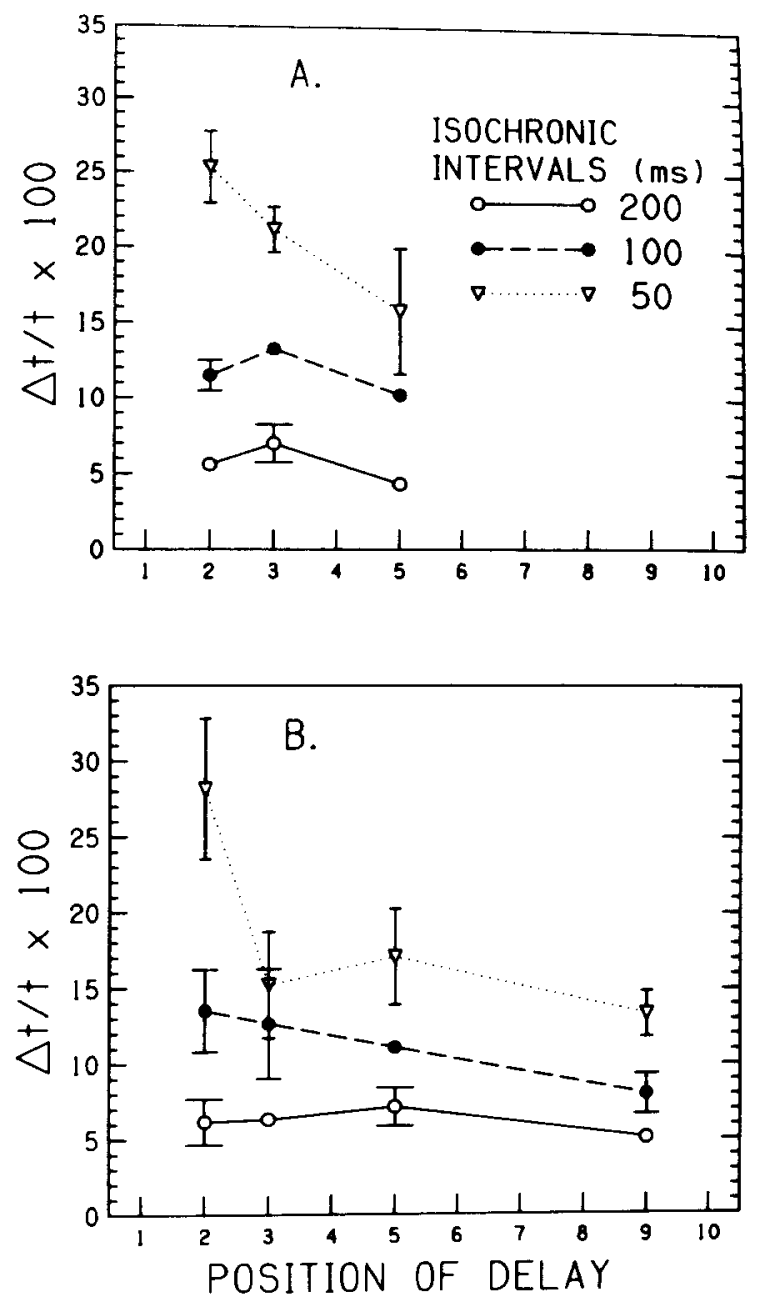

Figure 2. Relative difference limens (DLs; $\Delta t / t$ ) as a function of the position of the delayed tone in a pattern of 6 tones (A) or 10 tones (B). At an interonset interval (IOI) of $200 \mathrm{msec}$, the DL of about $6 \%$ is independent of position. At an 101 of $50 \mathrm{msec}$, the DL is generally greater and depends upon position, the best performance being for the delay at the end.

tempos, was the temporal position of the tone in the sequence. A more intrinsic tonal property, which may, according to the pilot studies, affect temporal discrimination, is frequency.

\section{Overview of the Design}

To assess the combined effects of a single pitch change and the position of delay of a single tone on the discriminability of deviation from isochrony, we employed isochronic series of 6 tones with IOIs of $200 \mathrm{msec}$ as standards. In any given sequence, 5 sinusoidal tones had a frequency of $1000 \mathrm{~Hz}$, while a sixth had a different frequency. All tones were $20 \mathrm{msec}$ in length. We used a complete repeated measures design with five factors: direction of the single frequency change (up or down from $1000 \mathrm{~Hz}$ ), size of the pitch interval (large or small), position of the tone with changed frequency (Position 2, 3, or 5), position of the delayed tone (Position 2, 3, or 5), and number of replications (three). The large frequency intervals $(1000 \mathrm{~Hz}$ up to $1667 \mathrm{~Hz}$ or $1000 \mathrm{~Hz}$ down to $600 \mathrm{~Hz}$ ) are called a "major sixth" and an "inverted minor third," respectively, in musical terms. They represent intervals in which the ratio of the higher to the lower frequency is 5:3. The smaller pitch intervals $(1000 \mathrm{~Hz}$ up to $1125 \mathrm{~Hz}$ or $1000 \mathrm{~Hz}$ down to $889 \mathrm{~Hz}$ ) are a "major second" or an "inverted minor seventh." These represent intervals in which the ratio of the higher to the lower frequency is $9: 8$.

\section{Method}

Apparatus. The apparatus was the same as that used in the first experiment. The computer collected subject responses and stored them in data files along with stimulus and timing parameters for each trial.

Subjects. The 3 subjects from Experiment 1 (H.D., S.K., and J.K.) were joined by J.B., who had had 5 years of musical experience. Both J.B. and J.K. were self-run subjects, and J.K. served as experimenter for most of the conditions for the other 2 subjects. All subjects were highly practiced and were paid for their services.

Procedure. The subjects performed 36 adaptive runs corresponding to the experimental conditions ( 2 directions $\times 2$ sizes $\times 3$ positions of pitch change $\times 3$ positions of tone delay $\times 3$ replications, in addition to a single run of the experiment as practice). The rest of the procedures were the same as in the previous experiment.

The 36 conditions of the experiment were presented in different random orders for each replication for each subject. Each subject completed all 36 conditions of any replication before beginning the next replication. The subjects completed the experiment in a period of about 3 weeks.

\section{Results}

A repeated measures ANOVA ( 2 pitch-change directions $\times 2$ change magnitudes $\times 3$ pitch-change positions $\times 3$ positions of delay $\times 3$ replications) was run on the estimates of the Weber ratio for discrimination of delay. No main effect or interaction involving replications was significant. Figure 3 shows discriminability of delay $(\Delta t / t$ $\times 100$ ) as a function of position of tone delay (abscissa) and position of pitch change (parameter) for each of the four combinations of pitch-change direction and size of interval. The standard-error bars in the figure represent variability across subjects and are based on $n=4$.

Table 1 shows the five significant interactions, which will be discussed in turn.

Pitch-change position $\times$ delay position. Table 2 shows this interaction, which is summed across the 2 directions $\times 2$ magnitudes of pitch change pictured in Figure 3 (summation is across all four panels). Poorer performance was found when the change of pitch occurred at the delay position. This result is not evident for tones in the third position, where performances were all poorer than the grand mean and were rather tightly bunched. Perhaps the third tone is affected by all pitch changes, whether at Position 2, 3, or 5, because the pitch-change and tone-delay positions are closer.

Direction $x$ interval size $\times$ pitch-change position. This interaction results from the fact that patterns with pitch changes at Tone 2 (circles in the panels of Figure 3) 

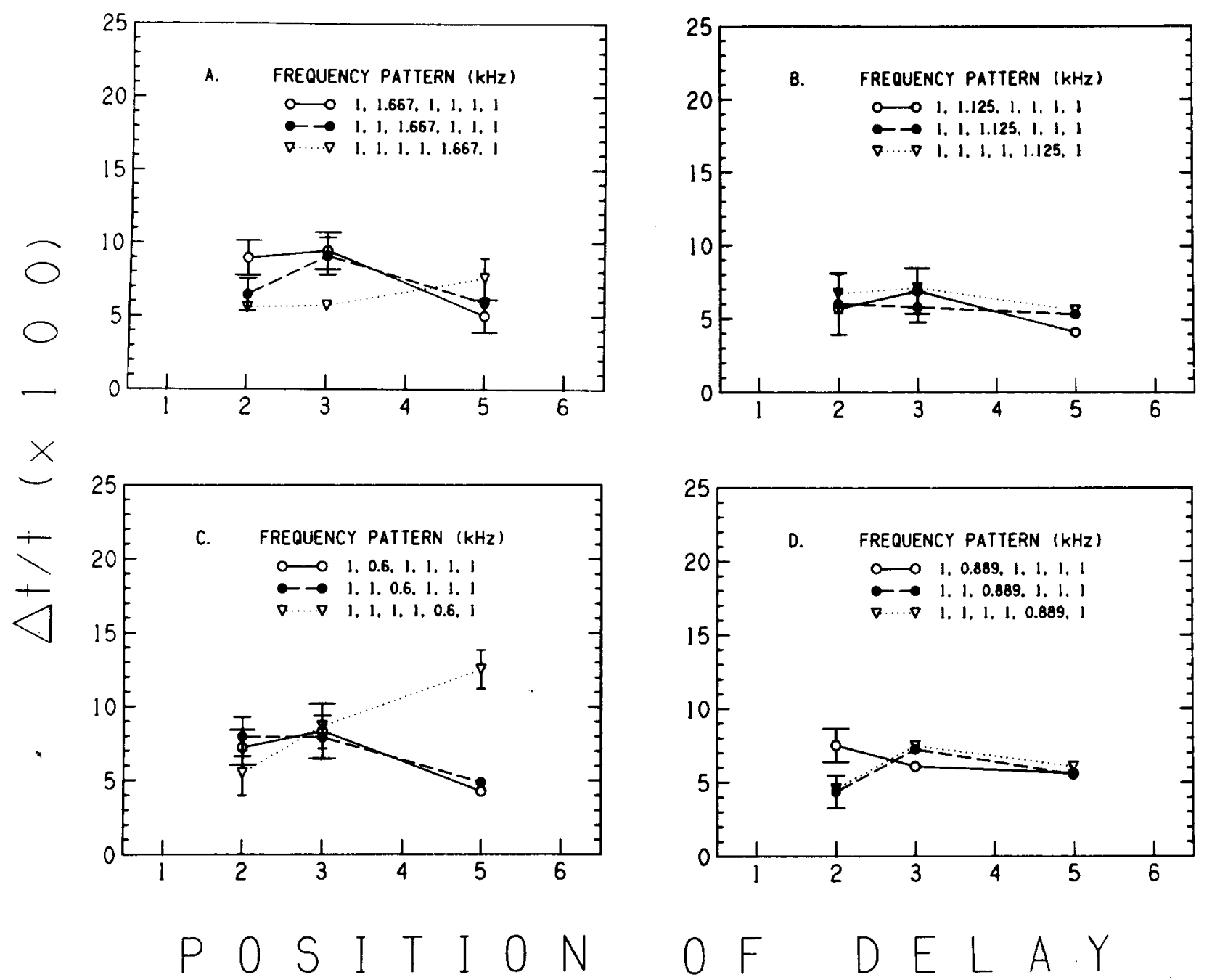

Figure 3. Relative difference limens (DLs) as a function of the delayed tone in a pattern of 6 tones, in one of which there was a pitch change, large upward in Panel A, small upward in Panel B, large downward in Panel C, and small downward in Panel D. Interonset interval was constant at $\mathbf{2 0 0}$ msec. There is a tendency for the DL to be relatively large when the delayed tone is just on or after the pitch change.

show the best performance averaged over delay positions when changes were upward and small (Panel B) or downward and large (Panel $C$ ), and the worst performance when pitch changes were upward and large (Panel A). On the other hand, patterns with pitch changes at Tone 5 (across all panels in Figure 3) show the reverse, with best performance for upward, large changes (Panel A) and poorer performance for upward, small changes (Panel B) or downward, large changes (Panel C). In the relation between the magnitude and direction of a pitch change, we may have shown a temporal effect related to the predominance of large up-going and small down-going pitch intervals in music (Vos \& Troost, 1989).

Direction $\times$ pitch-change position $\times$ delay position. This interaction results from the fact that downward pitch changes are generally more disruptive to performance than

Table 1

Significant Interactions of Positions of Pitch Change and Delay on Relative Difference Limen in Experiment 2

\begin{tabular}{llrl}
\hline \multicolumn{1}{c}{ Interaction } & \multicolumn{1}{c}{$d f$} & $F$ & $p$ \\
\hline Pitch change $\times$ delay position & 4,12 & 5.58 & .009 \\
Direction $\times$ interval size $\times$ changed pitch position & 2,6 & 21.22 & .002 \\
Direction $\times$ changed pitch position $\times$ delay position & 4,12 & 3.97 & .03 \\
Size $\times$ changed pitch position $\times$ delay position & 4,12 & 6.11 & .007 \\
Size $\times$ direction $\times$ changed pitch position $\times$ delay position & 4,12 & 6.19 & .007 \\
\hline
\end{tabular}


Table 2

Mean Values for Discriminability of Delay $(\Delta t / t \times 100)$ as a Function of Pitch-Change and Delay Positions

\begin{tabular}{ccccc}
\hline \multirow{2}{*}{$\begin{array}{c}\text { Position of } \\
\text { Pitch Change }\end{array}$} & \multicolumn{4}{c}{ Position of Delay } \\
\cline { 2 - 5 } & 2 & 3 & 5 & $M$ \\
\hline 2 & 7.4 & 7.7 & 4.7 & 6.6 \\
3 & 6.2 & 7.5 & 5.4 & 6.4 \\
5 & 5.6 & 7.3 & 8.0 & 6.9 \\
$M$ & 6.4 & 7.5 & 6.0 & 6.6 \\
\hline
\end{tabular}

Note-Means are summed over 2 magnitudes $\times 2$ directions of pitch change $\times 4$ subjects $\times 3$ replications per subject $=48$ measurements of $\Delta t / t$.

are upward pitch changes when the position of pitch change corresponds to delay positions, especially for Positions 2 and 5 (compare Panels $\mathrm{A}$ and $\mathrm{B}$ with Panels $\mathrm{C}$ and D of Figure 3 at Positions 2 and 5).

Size $\times$ pitch-change position $\times$ delay position. This interaction is due to the fact that large pitch changes are generally more disruptive to performance than are small changes when the position of pitch change corresponds to the delay position, again especially for Serial Positions 2 and 5 (compare Panels $A$ and $C$ with Panels $B$ and $D$ in Figure 3).

Size $\times$ direction $\times$ pitch-change position $\times$ delay position. Each combination of size and direction of pitch change represented in the four panels of Figure 3 is associated with a different ordering of the means for different positions of pitch change when either Tone 2 or Tone 3 was delayed. In addition, the combination of downward-large pitch change is associated with much poorer performance in detecting delays of Tone 5 than are the other combinations.

The pitch change represents an interruption in an otherwise isochronic, monotonic pattern. It may represent a demand for a switch in attention from one frequency channel to another (Divenyi \& Danner, 1977). In addition, the pitch change may be one method for introducing an accent in this homogeneous pattern. The accent is held to be a source of grouping in such patterns (Monahan \& Carterette, 1985; Monahan, Kendall, \& Carterette, 1987; Thomassen, 1982), so our study can be interpreted as one seeking to know whether temporal discrimination is more or less sensitive at or near points of accent. To further assess this accenting explanation, we turn to another source of accent: lengthening one of the intervals (Povel \& Okkerman, 1981).

\section{EXPERIMENT 3 SIMPLE NONISOCHRONIC MONOTONE PATTERNS}

In the next experiment, we expand our study of timing discrimination to include 6-tone series that have interonset intervals of two sizes: "long" (2), which is twice the length of "short" (1). Specifically, we were interested in whether timing DLs depend upon interval size within the pattern or on what might be called pattern regularity or simplicity (Povel \& Essens, 1985).

\section{Overview of the Design}

There were five patterns, each comprising a single, long interval and four short intervals: $21111,12111,11211$, 11121, and 11112. (After the sixth tone at the end of the fifth interval, the subjects reported that a sixth, unbounded interval appeared as "long.") The tone at each serial position, 2-6, was delayed for each of the five patterns at each of three tempos (where short was 50,100 , or $200 \mathrm{msec}$ ). This resulted in a complete factorial design of 5 patterns $\times 5$ positions of delay $\times 3$ tempos $(75$ conditions, total). All tones in these patterns were $1000-\mathrm{Hz}$ sinusoids, $20 \mathrm{msec}$ in length (2-msec rise-fall times and $16 \mathrm{msec}$ sustained).

Since we were also interested in the effect of tone duration within the intervals, we tested the 5 patterns $\times 5$ positions of delay $\times 2$ slower tempos with longer, $50-\mathrm{msec}$ tone durations (5-msec rise-fall times and $\mathbf{4 0} \mathrm{msec}$ sustained). This resulted in an additional 50 conditions, for a total of 125 conditions in the experiment.

All subjects were tested in each condition five times, the first replication was practice, and the last four were used for data analysis.

\section{Method}

Apparatus. The apparatus was the same as that described in the earlier experiments.

Subjects. There were 3 subjects, 1 male and 2 females. All subjects had normal audiograms. One subject (H.D.) was a dancer and had had 4 years of musical training. Another subject (C.S.) was a champion ice skater. C.S. was also a self-run subject and served as experimenter for most of the conditions for the other 2 subjects. Neither C.S. nor the 3rd subject (S.S.) had had any formal musical training. The subjects were paid for their services.

Procedure. In each replication of the experiment, the subjects performed 125 adaptive runs corresponding to the experimental conditions. The procedures were the same as in the previous experiments. Each run started with a delay of $25 \%$ of the preceding interval, well above the discrimination threshold. Thus, at the middle tempo, the initial trial with the 11211 pattern and delay of the third tone would take the form shown in Figure 4, although the order of the two comparisons might be reversed (sound-filled and silent portions are shown for each IOI in milliseconds).

The subjects received extensive training before data were taken. Each had an adaptive practice run of trials for each of the 125 conditions of the experiment. Each subject also had an additional practice run with each of the 25 fast-tempo conditions. The practice runs were performed over a period of 3-4 weeks. Then experimental data were taken over a period of 4 months, with daily sessions of about $2 \mathrm{~h}$ in which the subjects averaged about 18 adaptive runs per session.

Two subjects performed the 125 runs corresponding to the 125 conditions of the experiment in random order, while 1 had to be started on the slow tempo first. All were told or knew which pattern they were to hear and the place of the delay in the pattern before beginning a run. All subjects finished one replication of the experiment before starting the next.

\section{Results}

Two general analyses were performed, which were not independent of each other. In the first, a repeated measures ANOVA ( 3 tempos $\times 5$ positions of long interval $\times 5$ positions of delay $\times 4$ replications) was run on estimates of the DL for delay in milliseconds for the 75 con- 


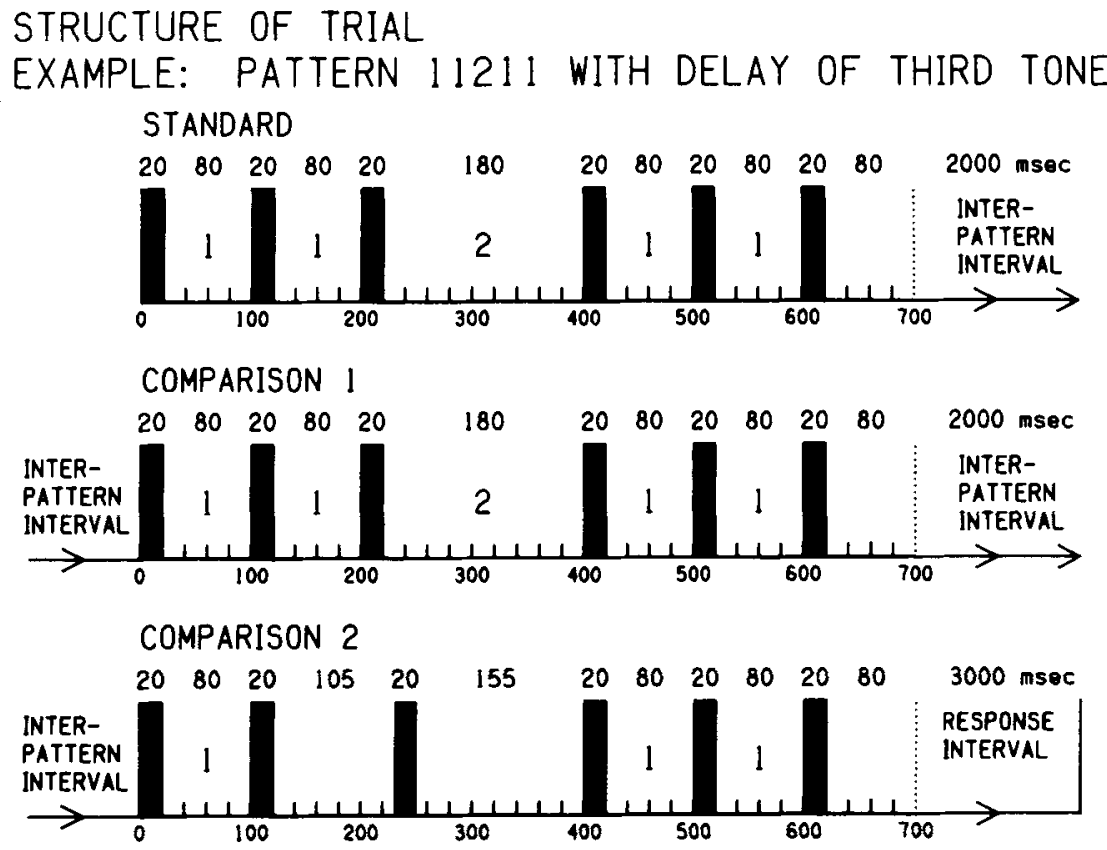

Figure 4. Timing diagram for Experiment 3.

ditions that employed 20-msec tones. All main effects except practice were significant, as shown in Table 3.

The means for each main effect, as well as levels of main effects that differ significantly from one another, are shown in Table 4. Post hoc analyses were NewmanKeuls tests with an alpha level of .05 for the whole experiment. Absolute DLs ( $\Delta t$ in milliseconds), averaged across positions of delay, were monotonically related to tempo-lowest for the fast tempo, which differed significantly from the two slower tempos. Absolute DLs were highest when the long interval was in Position 5, and were significantly higher when the delayed tone was in either Position 2 or Position 6; they were lowest for Tone 5. There were no interactions of replications (or practice) with any variable.

However, all of the remaining main effects are qualified by two- and three-way interactions. Figure 5, top row, shows the three-way interaction of serial position of the long interval (pattern shown by separations along abscissa) and position of the delayed tone, with tempo shown as the parameter. Each subject's performance was averaged over four replications for each experimental condition. Each point on the graphs of Figure 5 represents an average of these averages; the standard error for each point is thus based on an $n$ of 3 . Standard errors less than $1 \mathrm{msec}$ are not shown.

At the slowest tempo, and also to a great degree at the medium tempo, DLs were higher when the tone beginning or ending the long interval was delayed. In all conditions, DLs tended to be lower when the delayed tone marked the boundary between two shorter intervals. In addition, there was a tendency, especially at the medium tempo, for higher DLs when the delay occurred early in the pattern-that is, on the second tone. At all three tempos, DLs tended to be high when the final tone in the pattern was delayed-this was especially so when the long interval was in Position 5.

A second analysis was run to confirm the first and also to assess the effect of the variable of tone duration. In this analysis, the slow and medium tempos with 20 -msec

Table 3

Main Effects and Significant Interactions for Experiment 3

\begin{tabular}{lrrr}
\hline \multicolumn{1}{c}{ Effect } & $d f$ & $F$ & $p$ \\
\hline Tempo & 2,4 & 34.00 & .003 \\
Position of long interval & 4,8 & 8.96 & .005 \\
Position of delayed tone & 4,8 & 12.61 & .002 \\
Replications (practice) & 3,6 & 2.67 & .141 \\
Tempo X position of long interval & 8,16 & 3.25 & .009 \\
Position of long interval $\times$ position of delayed tone & 16,32 & 15.13 & .00001 \\
Tempo $\times$ position of long interval $\times$ position of delayed tone & 32,64 & 3.39 & .00001 \\
\hline
\end{tabular}


Table 4

Mean Difierence Limens (in msec) for Main Effects of Experiment 3

\begin{tabular}{ccc} 
& Tempo & \\
\hline Slow & Medium & Fast \\
\hline $13.92^{\mathrm{a}}$ & $11.98^{\mathrm{a}}$ & $7.55^{\mathrm{b}}$
\end{tabular}

\begin{tabular}{ccccc}
\multicolumn{5}{c}{ Position of Long Interval } \\
\hline 1 & 2 & 3 & 4 & 5 \\
\hline $10.94^{\mathrm{ab}}$ & $10.78^{\mathrm{ab}}$ & $10.47^{\mathrm{a}}$ & $10.82^{\mathrm{ab}}$ & $12.44^{\mathrm{b}}$
\end{tabular}

Position of Delayed Tone

\begin{tabular}{ccccc}
\hline 2 & 3 & 4 & 5 & 6 \\
\hline $12.65^{\mathrm{a}}$ & $10.61^{\mathrm{ab}}$ & $10.46^{\mathrm{ab}}$ & $8.96^{\mathrm{b}}$ & $13.06^{\mathrm{a}}$
\end{tabular}

\begin{tabular}{cccc}
\multicolumn{4}{c}{ Replications } \\
\hline 1 & 2 & 3 & 4 \\
\hline 11.51 & 11.47 & 10.87 & 10.74
\end{tabular}

Note-Within each row, means with different superscripts differ from each other $(p<.05)$ as measured by Newman-Keuls post hoc tests.

tone durations were compared with the same tempos with 50 -msec tone durations; in other words, the second analysis included all but the 25 conditions at the fast tempo. A repeated measures ANOVA $[2$ tempos $\times 5$ positions of the long interval $\times 5$ positions of delay $\times 4$ replications per subject $\times 2$ tone durations ( 20 or $50 \mathrm{msec}$ )] was run on estimates of DL in milliseconds.

The results of the second analysis are shown in Table 5 and are similar to those of the first analysis, with three exceptions. First, the listeners had lower DLs when the patterns were marked by tones that had $50 \mathrm{msec}$ filled with sound rather than only $20 \mathrm{msec}$, as shown in Figure 5 (lower panel) and in Figure 6; however, this effect only approached significance. Second, the main effect of tempo was not significant $(p<.06)$; but then there was no significant difference between slow and medium tempos in the first analysis. Third, the interaction of tempo with the position of the delayed tone was no longer significant; this reflects a similar tendency in both the slow- and the medium-tempo conditions to have heightened DLs at both the beginning and the end of the long interval.

The results shown in Figure 5 concern the absolute DL in milliseconds. Earlier figures had displayed the relative DL against the uniform base duration in an isochronic series. The calculation of a relative $\mathrm{DL}$ in the present experiment is not simple, since two different intervals might be used for a base, or denominator, of the Weber ratio. We show in Figure 6 a relative DL based on the shorter interval, the one that occurred most frequently in the pattern and might, therefore, be considered the "beat" of the pattern. In general, the sharper peaks of Figure 5, which are also the subject of the statistical analysis, are less evident. The curves are flatter (except for the clearly worse performance for the delay in the last position), and thus show less of a dependence on pattern (position of the long tone) and on delayed-tone position.

\section{Discussion}

The overall results between tempos do not follow Weber's law because DLs relative to the short interval $(\Delta t / t)$, as shown in Figure 6 for all experimental conditions, were generally lowest for the slowest tempo and highest for the fastest tempo. Here we are again finding evidence for the boundary where Weber's law begins to hold-which is apparently somewhere between 100 and $200 \mathrm{msec}$ (Killeen \& Weiss, 1987).

We interpret the results within patterns, especially in the slow-tempo conditions, as following Weber's law. A tone's delay at the boundary between two short intervals had a smaller absolute DL than did a delay between a short and a long interval or between a long and a short interval. The least noticeable delay occurred when the last tone of the sequence was delayed and when the interval before the final tone was long, which is similar to delaying the tone that is the boundary between two long intervals. We note that our results within patterns agree with the findings of Bharucha and Pryor (1986), who employed another procedure for investigating the discriminability of timing. They increased the length of single intervals by half a beat (and therefore lengthened the total pattern) in rhythmic patterns that comprised relatively random sequences of intervals. They found that absolute DLs for an increase were inversely related to the size of the increased interval. The size of temporal intervals in their experiment ranged from 200 to $1,200 \mathrm{msec}$.

\section{CONCLUSIONS}

Listeners can discriminate an interval between two brief sounds with great precision-changes in the size of the interval of the order of $5 \%-10 \%$ can be noticed. This statement is generally true for intervals down to about

Table 5

Main Effects and Significant Interactions for Experiment 3 (Second Analysis)

\begin{tabular}{lccc}
\hline \multicolumn{1}{c}{ Effect } & $d f$ & \multicolumn{1}{c}{$\boldsymbol{F}$} & $p$ \\
\hline Tempo & 1,2 & 17.16 & .054 \\
Position of long interval & 4,8 & 8.16 & .006 \\
Position of delayed tone & 4,8 & 7.08 & .009 \\
Replications (practice) & 3,6 & 3.76 & .07 \\
Length of tones (20 vs. $50 \mathrm{msec})$ & 1,2 & 14.80 & .061 \\
Position of long interval $\times$ position of delayed tone & 16,32 & 15.44 & .0001 \\
Tempo $\times$ position of long interval $\times$ position of delayed tone & 32,64 & 3.63 & .0009 \\
\hline
\end{tabular}



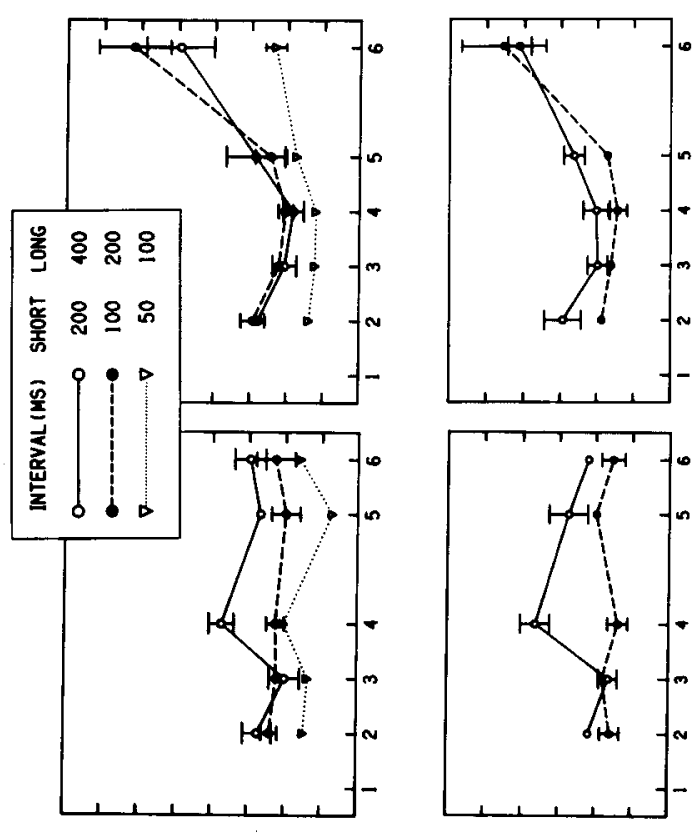

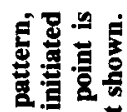

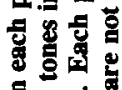

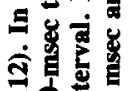

寻家-

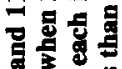

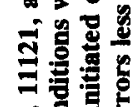

$=8$

省多

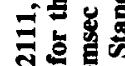

考它客

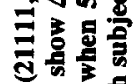

$z$
0
5
1
5
0
0
0
$w$
$z$

\&

范要
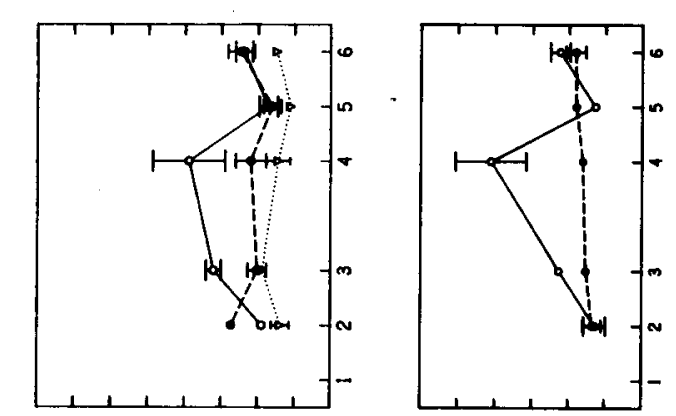

영

递官

量

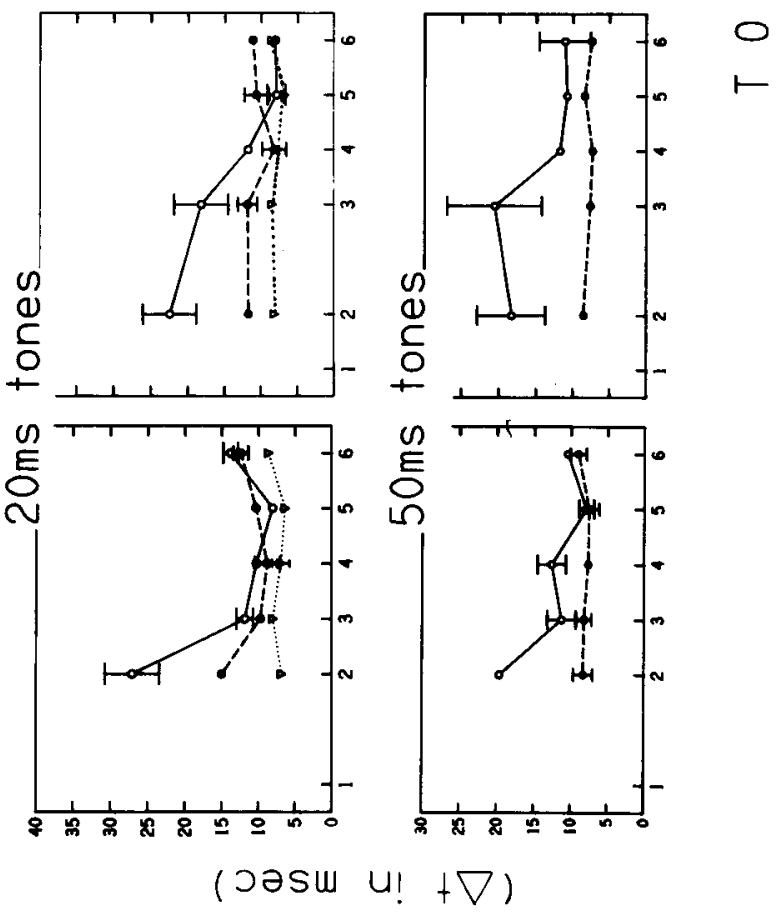

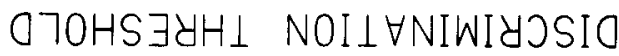

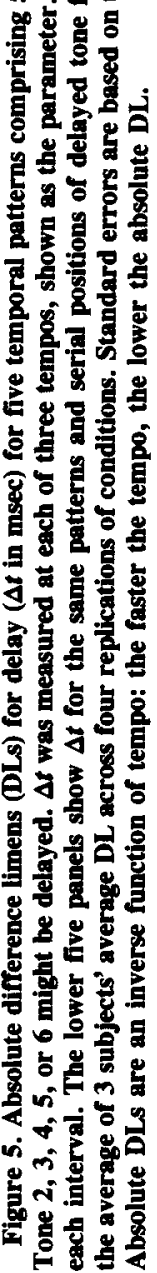




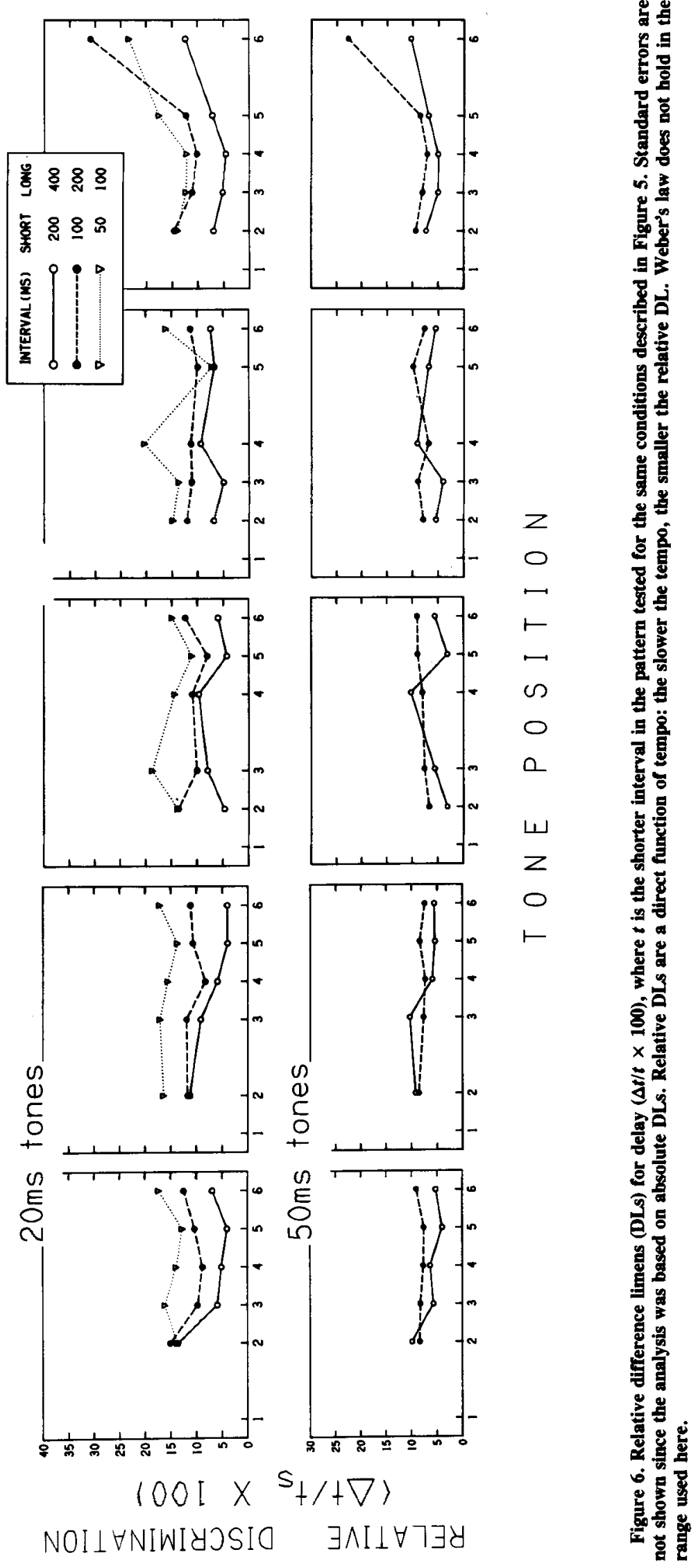


100 msec. Furthermore, even when a sequence of such intervals is presented, the discrimination remains equally good independent of its position in the sequence-certainly for intervals of $200 \mathrm{msec}$, and nearly so for intervals of $100 \mathrm{msec}$. Smaller intervals of $50 \mathrm{msec}$ show poorer discrimination and, in the case of a sequence of such intervals, a marked dependence on position in the pattern, the best discrimination being near the end of the sequence.

When one introduces into a sequence of tones a pitch change in one of them, precision of temporal discrimination is somewhat poorer for the pitch-changed tone, which now appears to be accented. Similar observations are made in the case where, instead of a pitch change, an interval change is made, such that instead of isochronicity, the sequences exhibit a kind of accent by virtue of having one interval duration being twice as long as all the others. Here again, precision of temporal discrimination is worse around the long interval. It is not clear whether this poorer performance is related to an interval that is different, that is longer, that constitutes the basis of an accent, or is some combination.

\section{REFERENCES}

ABEL, S. M. (1972). Duration discrimination of noise and tone bursts. Journal of the Acoustical Society of America, 51, 1219-1223.

Bharucha, J. J., \& PrYoR, J. H. (1986). Disrupting the isochrony underlying rhythm: An asymmetry in discrimination. Perception \& Psychophysics, 40, 137-141.

Creelman, C. D. (1962). Human discrimination of auditory duration. Joumal of the Acoustical Society of America, 34, 582-593.

Divenyi, P. L., Danner, W. F. (1977). Discrimination of time intervals marked by brief acoustic pulses of various intensities and spectra. Perception \& Psychophysics, 21, 125-142.

Fitzgibbons, P. J., Pollatsek, A., \& Thomas, I. B. (1974). Detection of temporal gaps within and between perceptual tonal groups. Perception \& Psychophysics, 16, 522-528.

FRY, D. B. (1955). Duration and intensity as physical correlates of linguistic stress. Journal of the Acoustical Society of America, 35, 765-769.

HenRY, F. M. (1948). Discrimination of the duration of a sound. Journal of Experimental Psychology, 45, 734-743.
HIRSH, I. J. (1987). Timing in auditory perception. Journal of the Acoustical Society of America, 81(Suppl. 1), $\$ 90$.

KILLEEN, P. R., \& WeISS, N. A. (1987). Optimal timing and the Weber function. Psychological Review, 94, 455-468.

Monahan, C. B., \& Carterette, E. C. (1985). Pitch and duration as determinants of musical space. Music Perception, 3, 1-32.

Monahan, C. B., Kendall, R. A., \& Carterette, E. C. (1987). The effect of melodic and temporal contour on recognition memory for pitch change. Perception \& Psychophysics, 41, 576-600.

PollaCK, I. (1967). Asynchrony: The perception of temporal gaps within auditory pulse patterns. Joumal of the Acoustical Society of America, 42, 1335-1340.

Povel, D.-J., \& Essens, P. (1985). Perception of temporal patterns. Music Perception, 2, 411-440.

Povel, D.-J., \& OKKerman, H. (1981). Accents in equitone sequences. Perception \& Psychophysics, 30, 565-572.

Small, A. M., \& Campbell, R. A. (1962). Temporal differential sensitivity for auditory stimuli. American Journal of Psychology, 75, 401-410.

SORKIN, R. D. (1987). Temporal factors in the discrimination of tonal sequences. Journal of the Acoustical Society of America, 82, 1218-1226.

SorKIN, R. D., BogGs, G. J., \& BrADY, S. L. (1982). Discrimination of temporal jitter in patterned sequences of tones. Journal of $E x$ perimental Psychology: Human Perception \& Performance, 8, 46-57.

Thomassen, J. M. (1982). Melodic accent: Experiments and a tentative model. Journal of the Acoustical Society of America, 71, 1596-1605.

UMEDA, N. (1975). Vowel duration in American English. Journal of the Acoustical Society of America, 58, 434-445.

Vos, P. G. (1977). Temporal duration factors in the perception of auditory rhythmic patterns. Scientific Aesthetics/Sciences de l'An, 1, 183-199.

Vos, P. G., \& Troost, J. M. (1989). Ascending and descending melodic intervals: Statistical findings and their perceptual relevance. Music Perception, 6, 383-396.

Watson, C. S., Wroton, H. W., Kelly, W. J., \& Benbassat, C. A. (1975). Factors in the discrimination of tonal patterns: 1 . Component frequency, temporal position and silent intervals. Journal of the Acoustical Society of America, 57, 1175-1185.

Woodrow, H. (1951). Time perception. In S. S. Stevens (Ed.), Handbook of experimental psychology (pp. 1224-1236). New York: Wiley.

(Manuscript received January 13, 1989; revision accepted for publication September 11, 1989.) 